\title{
Correction to: Pineal Neurosurgery
}

Samer S. Hoz, Ali A. Dolachee, Hayder R. Salih, Zaid S. Aljuboori, Wisam D. Selbi, Giath Al-Dayri, and Mohammed Maan Al-Salihi

\section{Correction to: S. S. Hoz et al. (eds.), Pineal Neurosurgery, https://doi.org/10.1007/978-3-030-53191-1}

The original version of the book was revised: Affiliation of one of the editors, Mohammed Maan Al-Salihi, has been updated. The erratum to the book is available at https://doi.org/10.1007/978-3-030-53191-1_11 